ISSN 0103-9954

\title{
AVALIAÇÃO DO POTENCIAL DE APROVEITAMENTO ENERGÉTICO DOS RESÍDUOS DE MADEIRA E DERIVADOS GERADOS EM FÁBRICAS DO POLO MOVELEIRO DE UBÁ - MG
}

\author{
EVALUATION OF ENERGY USE POTENTIAL OF WOOD RESIDUES AND DERIVATIVES \\ GENERATED IN THE INDUSTRIAL FURNITURE STAVE OF UBÁ - MINAS GERAIS STATE
}

\author{
Rogério Machado Pinto Farage ${ }^{1}$ Ana Augusta Passos Rezende ${ }^{2}$ \\ Cláudio Mudado Silva ${ }^{3}$ Wiliam Gomes Nunes ${ }^{4}$ Angélica de Cássia Oliveria Carneiro ${ }^{5}$ \\ Danielle Biajoli Vieira ${ }^{6}$ Cleuber Lúcio Silva Rodrigues ${ }^{7}$
}

\begin{abstract}
RESUMO
O presente estudo avaliou o potencial de reaproveitamento energético dos resíduos de madeira e seus derivados gerados no Polo Moveleiro de Ubá, MG. Painéis reconstituídos de MDF (medium density fiberboard), aglomerado e compensado foram caracterizados e classificados, identificando assim as oportunidades e possíveis limitações quanto à sua utilização. Por meio de diagnóstico realizado em 11 fábricas de móveis, verificou-se que os resíduos de madeira compreendem mais de $90 \%$ do total dos resíduos sólidos gerados. Além da grande quantidade gerada, não foi identificada nenhuma ação integrada entre as fábricas do Polo para o adequado gerenciamento dos resíduos, negligenciando seus riscos ambientais e sanitários, bem como seu potencial energético. Contudo, os teores de umidade e poder calorífico dos resíduos demonstraram potencial para o seu reaproveitamento energético através da produção de briquetes. Os gases gerados em ensaios de combustão dos resíduos não apresentaram substâncias ou compostos tóxicos acima dos limites preconizados pelas normas ambientais, verificando, neste aspecto, um bom desempenho ambiental para o aproveitamento destes resíduos conforme proposto pelo presente estudo. Entretanto, as cinzas dos resíduos de aglomerado BP (baixa pressão) e FF (finish foil) apresentaram elevadas concentrações de cromo, enquadrando-se como Classe I (perigosos), segundo a ABNT/NBR 10004/2004.
\end{abstract}

Palavras-chave: fábricas de móveis; resíduos de madeira; aproveitamento energético.

\begin{abstract}
The present study evaluated the energy reuse potential of the wooden residues and its derivatives generated in the industrial furniture stave of Ubá, Minas Gerais state, Brazil. Reconstituted panels of MDF (medium density fiberboard), particleboards and plywood were characterized and classified. A research carried out in 11 furniture plants verified that the wooden residues represent more than $90 \%$ of the solid wastes. Besides of this the large amount generated, no integrated action was identified to the management of the residues, neglecting its environmental and sanitary risks, as well as its energy use potential. However, the moisture and calorific power of the residues demonstrated a high potential energy use through the

1. Administrador, Mestre em Engenharia Ambiental, Instituto de Ciências Exatas e Biológicas, Universidade Federal de Ouro Preto, Morro do Cruzeiro, CEP 35400-000, Ouro Preto (MG). rogeriompf@gmail.com

2. Engenheira Civil, Dr ${ }^{\mathrm{a}}$., Professora Adjunta, Departamento de Engenharia Civil, Centro de Ciências Exatas, Universidade Federal de Viçosa, Av. P.H. Rolfs, s/n, CEP 36570-900, Viçosa (MG). aaprezende@yahoo.com.br

3. Engenheiro Civil, Dr., Professor Adjunto, Departamento de Engenharia Florestal, Centro de Ciências Agrárias, Universidade Federal de Viçosa, Av. P.H. Rolfs, s/n, CEP 36570-900, Viçosa (MG). mudado@ufv.br

4. Engenheiro Ambiental, Graduado em Engenharia Ambiental pela Universidade Federal de Viçosa, Av. P.H. Rolfs, s/n, CEP 36570-900, Viçosa (MG). wilgones@yahoo.com.br

5. Engenheira Florestal, $\mathrm{Dr}^{\mathrm{a}}$., Professora Adjunta, Departamento de Engenharia Florestal, Centro de Ciências Agrárias, Universidade Federal de Viçosa, Av. P.H. Rolfs, s/n, CEP 36570-900, Viçosa (MG). cassiacarneiro@ufv.br

6. Engenheira Ambiental, Graduada em Engenharia Ambiental pela Universidade Federal de Viçosa, Av. P.H. Rolfs, s/n, CEP 36570-900, Viçosa (MG). danibiajoli@yahoo.com.br

7. Engenheiro Ambiental, Graduado em Engenharia Ambiental pela Universidade Federal de Viçosa, Av. P.H. Rolfs, s/n, CEP 36570-900, Viçosa (MG). clsrodrigues@yahoo.com.br

Recebido para publicação em 20/05/2009 e aceito em 16/12/2011
\end{abstract}


briquette production. The gases generated by combustion of the residues did not presented toxic substances above the limits of Brazilian legislation. However, the leached ashes of the residues of agglomerated BP (low pressure) and FF (finish foil) presented high chromium concentrations, classifying them as Class I (dangerous), according to ABNT/NBR 10004/2004.

Keywords: furniture production; wooden residues; energy use.

\section{INTRODUÇÃO}

Os resíduos sólidos representam um dos grandes desafios previstos para o século XXI. Essa preocupação se justifica pelo crescente aumento da sua geração e pelo reconhecido déficit de soluções sanitárias e ambientalmente adequadas à sua disposição final ou reaproveitamento, sendo estas últimas ainda incipientes no país, embora de grande potencial. Além do aumento na quantidade gerada, são descartados, diariamente no ambiente, resíduos de composições cada vez mais complexas, tornando ainda mais difíceis e onerosos os processos de reaproveitamento, limitando a capacidade de sua assimilação pelo ambiente natural. Somada aos impactos sanitários e ambientais causados pela sua disposição inadequada, a geração de resíduos retrata um grande desperdício de matéria-prima e energia. Dentro deste panorama, as indústrias de transformação são responsáveis pelos maiores impactos ambientais registrados.

Segundo estimativas da Associação Brasileira de Empresas de Limpeza Pública e Resíduos Especiais (ABRELPE, 2007), através de diagnóstico realizado em 10 Unidades Federativas (AC, AP, CE, GO, MG, PE, RS, PR, RJ e SP), os resíduos sólidos de origem industrial somam cerca de 86,5 milhões de toneladas/ano.

O setor moveleiro representa expressiva importância socioeconômica para o Brasil, notadamente pelo intensivo emprego de mão de obra. De acordo com a Associação Brasileira das Indústrias do Mobiliário (ABIMOVEL, 2005), o setor moveleiro no Brasil é formado por aproximadamente 16.000 fábricas, segmentado em polos regionais, dos quais se destacam os polos do Rio Grande do Sul (Polo de Bento Gonçalves), Santa Catarina (Polo de São Bento do Sul), Paraná (Polo de Arapongas), São Paulo (Polo de Votuporanga), Espírito Santo (Polo de Linhares) e Minas Gerais (Polo de Ubá).

O Polo Moveleiro de Ubá é formado por cerca de 400 fábricas de móveis, distribuídas em 9 municípios do Estado de Minas Gerais, localizados na microrregião de Ubá. Este Polo representa uma fonte de economia estratégica para a região, gerando cerca de 20.000 empregos diretos e indiretos (FIEMG, 2008).

Paralelo à sua importância socioeconômica, as fábricas do Polo Moveleiro de Ubá trazem consigo um expressivo passivo ambiental, principalmente devido ao descarte inadequado de resíduos sólidos, especialmente de madeira e seus derivados (painéis de aglomerados, MDF e compensados), representando mais de $90 \%$ do total de resíduos gerados. Os resíduos dos painéis reconstituídos sugerem uma preocupação maior, devido à presença dos adesivos, geralmente ureiaformaldeído ou fenol-formaldeído, presentes na sua composição.

Silva et al. (2005) realizaram um diagnóstico em 55 fábricas de móveis no Polo Moveleiro de Ubá, identificando uma grande deficiência em relação ao controle e gerenciamento destes resíduos. Constataram que a disposição dos resíduos era feita aleatoriamente, a exemplo da sua queima a céu aberto e doação ou venda a terceiros, sem nenhum controle sobre quantidade e aplicação final. Situação semelhante foi identificada por Schneider et al. (2003), a partir de estudo realizado com 26 fábricas do Polo Moveleiro de Bento Gonçalves - RS, onde cerca de $53 \%$ dos resíduos de madeira gerados eram vendidos a terceiros geralmente sem conhecimento da sua destinação final.

Abreu et al. (2009) propõem o reaproveitamento dos resíduos de painéis de madeira na forma de aparas, através da produção de pequenas peças artesanais. De acordo com Silva et al. (2005), dentre as formas de reaproveitamento dos resíduos de madeira e derivados foram verificadas sua utilização como forração de cama de frango em granjas, queima em fornos e caldeiras, e ainda como condicionador de solo. Nota-se que essas formas de reaproveitamento e disposição, além de apresentar possíveis riscos ao ambiente natural e à saúde pública, desconsideram o grande potencial destes resíduos como fontes de matéria-prima para geração de energia. Segundo os autores, esse diagnóstico refletiu uma situação de risco para o Polo Moveleiro de Ubá, não somente em relação aos problemas 
ambientais e sanitários, como os socioeconômicos, visto que as fábricas têm sofrido incessante pressão dos órgãos ambientais competentes devido às formas inadequadas para com o gerenciamento e controle de suas fontes poluidoras, colocando em risco o seu desenvolvimento.

O presente estudo foi motivado pela demanda urgente de tecnologias e processos de controle e gerenciamento que permitam reaproveitamento e disposição final adequada dos resíduos de madeira e seus derivados pelas fábricas de móveis do Polo de Ubá. Somado a isso, a crescente demanda energética mundial associada ao apelo pelas chamadas energias limpas e renováveis, sugerem uma grande oportunidade por meio do reaproveitamento energético destes resíduos.

$\mathrm{O}$ reaproveitamento dos resíduos de madeira e derivados para fins de geração de energia foi previsto na forma de briquetes, produto resultante da compactação dos resíduos de madeira sob alta pressão e temperatura. Segundo Quirino (2002), entre as vantagens do briquete destacase sua alta densidade, dimensões padronizadas e baixa umidade, barateando sobremaneira os custos de transporte e armazenagem deste produto. Cabe destacar ainda seu elevado peso específico (cerca de $1.200 \mathrm{kgm}^{-}{ }^{3}$ ), resultando em um produto com concentração de poder calorífico por volume bem superior ao dos resíduos supracitados e da própria lenha.

Pires et al. (2008) realizaram um estudo da viabilidade econômico-financeira da implantação de uma central de tratamento dos resíduos gerados pelas fábricas de móveis do Polo Moveleiro de Ubá, prevendo a comercialização destes para fins de reciclagem, matéria-prima para outros processos e reaproveitamento energético, identificando viabilidade financeira para o empreendimento. Cabe aqui destacar a venda dos briquetes, responsável por $88 \%$ do total das receitas previstas.

O objetivo da presente pesquisa foi estudar o potencial de reaproveitamento de resíduos de madeira e derivados de madeira gerados em indústrias de móveis do Polo Moveleiro de Ubá para fins de geração de energia, em seus aspectos técnicos e ambientais, abrangendo a:

- quantificação e caracterização físicoquímica dos resíduos;

- classificação dos resíduos quanto à sua periculosidade;

- análise química dos gases gerados pela queima dos resíduos;
- classificação das cinzas resultantes da queima dos resíduos.

\section{MATERIAL E MÉTODOS}

\section{Objeto de estudo}

O trabalho foi realizado em parceria com 11 fábricas de móveis do Polo Moveleiro de Ubá, Minas Gerais. Estas fábricas foram escolhidas aleatoriamente, tendo como pré-requisito a fabricação de móveis de madeira. Conforme classificação segundo o porte, definida pelo Serviço Brasileiro de Apoio às Micro e Pequenas Empresas (SEBRAE), compreendem 1 micro, 4 pequenas, 5 médias e 1 grande empresa.

\section{Quantificação e caracterização dos resíduos de madeira e seus derivados}

A identificação dos resíduos foi realizada por meio da aplicação de um Inventário de Resíduos Sólidos Industriais, elaborado conforme orientação do Conselho Nacional de Meio Ambiente - CONAMA, através da Resolução $n^{\circ}$ 313/2002 (CONAMA, 2002). Sua aplicação foi realizada de forma presencial, por meio de visitas técnicas às 11 fábricas de móveis, no período de outubro a dezembro de 2006.

\section{Amostragem}

Os resíduos amostrados para as análises físico-químicas se dividiram em 2 grupos distintos, conforme apresentado na Tabela 1.

O Grupo A é constituído por resíduos compostos de diversas matérias-primas, nas formas de serragem e pó de madeira. A coleta e o acondicionamento destes resíduos nas fábricas em estudo ocorrem por meio de um sistema de exaustão composto por tubulações conectadas aos equipamentos onde são gerados, direcionando os mesmos, que são misturados, a um silo localizado na área externa do galpão fabril não ocorrendo sua segregação no processo fabril. As amostras de resíduos do Grupo A foram coletadas junto aos silos de armazenagem. Estas coletas foram realizadas mediante a abertura dos silos (suspensos), procedendo à amostragem de pontos diversos da massa de resíduos conforme os grupos apresentados na Tabela 1.

Os resíduos do Grupo B referem-se aos de madeira e seus derivados na forma de aparas (pedaços de madeiras de dimensões variadas). $\mathrm{O}$ acondicionamento destes resíduos é feito em 
TABELA 1: Grupos de resíduos amostrados.

TABLE 1: Group of sampled wood wastes.

\begin{tabular}{|c|c|}
\hline Grupo A (serragem e pó) & Grupo B (aparas) \\
\hline $\begin{array}{l}\text { A1-mistura de resíduos de painéis de MDF, aglomerado, } \\
\text { compensado s/ revestimento e madeira maciça }\end{array}$ & B1 - madeira maciça \\
\hline A2-resíduo de madeira maciça & B2 - compensado \\
\hline A3-resíduo de MDF (medium density fiberboard) & B3 - MDF (medium density fiberboard) \\
\hline $\begin{array}{l}\text { A4-mistura de resíduos de painéis de MDF, aglomerado e } \\
\text { compensado com e s/ revestimento }\end{array}$ & B4 - aglomerado s/ revestimento \\
\hline $\begin{array}{l}\text { A5-mistura de resíduos de painéis de MDF, aglomerado e } \\
\text { compensado s/ revestimento }\end{array}$ & B5 - aglomerado revestido BP (baixa pressão) \\
\hline $\begin{array}{l}\text { A6-pó de lixação de painéis (MDF, aglomerado e } \\
\text { compensado) }\end{array}$ & tido FF (finish foil) \\
\hline
\end{tabular}

tambores, caixotes de madeira, caçambas e/ ou contêineres, permitindo sua segregação por matéria-prima de origem no momento da geração. As amostras do Grupo B foram coletas junto a caçambas, tambores e caixotes, locais comuns de armazenagem destes resíduos nas fábricas amostradas. Estas amostras foram coletadas por funcionários previamente designados das respectivas empresas inventariadas, devidamente orientados para tal. As amostras coletadas foram acondicionadas em sacos plásticos transparentes, de 50 litros.

A determinação de amostras de resíduos do Grupo A permitiu estudos específicos do potencial energético e riscos ambientais relacionados ao reaproveitamento dos resíduos formados pela atual forma de acondicionamento nas fábricas, refletindo os condicionantes da situação real. As amostras de resíduos do Grupo B foram estabelecidas com o objetivo de avaliar os potenciais e riscos de cada tipo de resíduo (classificados quanto às suas matérias-primas).

\section{Caracterização físico-química dos resíduos de madeira e seus derivados}

As análises físico-químicas dos resíduos de madeira e seus derivados foram realizadas para a avaliação do potencial de reaproveitamento energético destes resíduos.

\section{Teor de umidade e cinzas}

As determinações dos teores de umidade e cinzas foram realizadas segundo orientação das normas NBR 8293/1983 e NBR 8289/1983, respectivamente, em três repetições.

\section{Poder calorífico}

A determinação do poder calorífico dos resíduos amostrados procedeu-se conforme a norma American Society for Testing Materials ASTM D-2015-66, em 3 repetições por amostra de resíduos. O preparo das amostras se deu por meio do refinamento dos resíduos em um moinho Thomas Wiley - modelo 4, e o seu peneiramento em peneiras de aço inox de 35 mesh (abertura 0,425 mm) e 60 mesh (abertura $0,251 \mathrm{~mm}$ ). Os ensaios foram realizados em um Calorímetro Adiabático.

\section{Densidade aparente da serragem e do pó}

$O$ cálculo da densidade aparente baseiase na relação entre massa e volume $\left(\mathrm{kg} / \mathrm{m}^{3}\right)$. Neste caso utilizou-se uma proveta de $500 \mathrm{ml}$ (volume conhecido). As amostras dos resíduos foram acondicionadas na proveta sem nenhum procedimento de compactação. Foram realizadas 5 repetições por amostra de resíduo. Este teste foi realizado para as amostras de resíduos do Grupo A.

\section{Classificação dos resíduos quanto à sua periculosidade}

A classificação dos resíduos quanto à sua periculosidade se deu por meio da adoção das normas da Associação Brasileira de Normas Técnicas NBR 10004, 10005 e 10006 (ABNT, 2004). Foram classificados os resíduos amostrados, como também as cinzas procedentes da queima das respectivas amostras.

Nos testes de lixiviação, realizados conforme a NBR 10.005 para classificação de perigoso (classe I) ou não, os parâmetros orgânicos foram determinados apenas para as amostras B1, B2, B3, B4, B5, B6 e A6. Não se caracterizou as amostras A1 a A5 por considerar que as mesmas estão representadas nas amostras do grupo B. Os parâmetros inorgânicos foram determinados para os extratos lixiviados de todas as amostras de resíduos 
dos Grupos A e B.

\section{Parâmetros orgânicos}

- Compostos Orgânicos Voláteis (VOC): cloreto de vinila, 1,1-Dicloroeteno, 1,2-Dicloroetano, Tetracloreto de carbono, Benzeno, Tricloroeteno, Tetracloroeteno, Clorobenzeno, 1,4-Diclorobenzeno, Hexaclorobutadieno, Metiletilcetona, Tolueno, Acetona e Clorofórmio.

- Compostos Orgânicos Semivoláteis (SVOC): Fenol, 2-Metilfenol, 3-Metilfenol, 4-Metilfenol, 2-Clorofenol, 2,4-Dimetilfenol, 2,6-Diclorofenol, 2,4-Diclorofenol, 2-Nitrofenol, 2,4,5-Triclorofenol, 2,3,4,6-Tetraclorofenol, Pentaclorofenol, 2,4-Dinitrotolueno e Anilina.

- Formaldeído.

\section{Parâmetros inorgânicos} e Cromo.

- Bário, Arsênio, Chumbo, Selênio, Cádmio

As análises dos parâmetros orgânicos do extrato lixiviado das amostras seguiram os métodos US EPA 8315A (US EPA, 1996) e PE4.9-127 Rev.6 (ANALYTICAL SOLUTIONS S.A., 2006).

Para os parâmetros inorgânicos, o preparo do extrato lixiviado das amostras de resíduos e a leitura dos parâmetros foram realizados, respectivamente, no Laboratório de Celulose e Papel e no Laboratório de Solos da Universidade Federal de Viçosa (UFV).

\section{Análise dos gases gerados na queima dos resíduos de madeira e seus derivados}

As emissões gasosas geradas durante o processo de combustão dos resíduos foram analisadas segundo os parâmetros: Formaldeido, Clorofórmio, $\mathrm{NO}_{2}, \mathrm{CO}_{2}$ e CO. Na definição destes parâmetros considerou-se a relevância das substâncias e compostos identificados nas análises químicas do extrato de lixiviação e das características inerentes às matérias-primas de origem dos resíduos analisados. Além disso, as referidas considerações foram confrontadas com a Resolução 382 do Conselho Nacional de Meio Ambiente (CONAMA, 2006). Os resíduos para os quais as emissões gasosas foram analisadas compreenderam: B1 (madeira maciça), B3 (MDF) e B5 (Aglomerado BP).

$\mathrm{O}$ processo de queima da biomassa (resíduos) foi realizado em um equipamento onde os resíduos de madeira, na forma de serragem e pó, foram introduzidos através de um compartimento de armazenagem (espécie de silo horizontal), provido de uma rosca sem-fim responsável pela condução do material até a câmara de combustão em intervalos intermitentes, controlados manualmente. A câmara de combustão é composta por um sistema de injeção de ar contínuo. Foi projetada e instalada uma chaminé na extremidade da câmara de combustão do equipamento. Essa chaminé se prolonga horizontalmente $80 \mathrm{~cm}$, curvando-se verticalmente $90^{\circ}$ e se prolongando por mais 1 metro. A coleta dos gases se deu por meio de um orifício de $8 \mathrm{~cm}$ de diâmetro no corpo da chaminé, a uma altura de $80 \mathrm{~cm}$.

O equipamento utilizado para a coleta dos gases foi um Coletor Isocinético de Poluentes Atmosféricos - CIPA 0112, do tipo diafragma, em conjunto com placa de orifício, e um tubo Pitot em "S".

A análise dos gases foi realizada por Cromatógrafo Gasoso com detector de FID (Detector de Ionização de Chama) dotado de coluna semicapilar.

Durante a queima dos resíduos B1 e B5, a temperatura média foi de 520 a $580{ }^{\circ} \mathrm{C}$. Para a amostra B3, esta temperatura se manteve entre 351 e $354^{\circ} \mathrm{C}$.

As amostragens e análises foram realizadas conforme as seguintes metodologias e normas para dutos e chaminés de fontes estacionárias: NBR 8969 (ABNT, 1985); NBR 12020 (ABNT, 1992); NBR 10700 (ABNT, 1989); NBR 10701 (ABNT, 1989); NBR 10702 (ABNT, 1989);NBR11966(ABNT,1989);NBR11967(ABNT, 1990); NBR 12019 (ABNT, 1990); Método adaptado para absorção de Formaldeído em solução de DNPH (2,4-Dinitrofenilidrazina); CETESB L9.229 (CETESB, 1992); EPA Method 030 (US EPA, 1986).

\section{RESULTADOS E DISCUSSÃO}

O inventário de resíduos sólidos possibilitou identificar os tipos e quantidades de resíduos gerados, assim como os seus respectivos pontos de geração e formas de acondicionamento. Os principais resíduos diagnosticados foram: resíduos de madeira maciça, painéis de aglomerado crus e revestidos (BP e FF), painéis de $\mathrm{MDF}$, e painéis de compensado. Os resíduos de madeira maciça constituem-se essencialmente por madeira de espécies Pinus sp., Eucalyptus, Couratari spp.. (Tauari ou Embirema). Os resíduos apresentaram-se na forma de aparas, serragem e pó de madeira. 
A geração dos resíduos de madeira e seus derivados foram verificados nas etapas de corte (aparas, cavacos e pó de madeira), usinagem e desempeno (serragem e pó de madeira), furação (serragem e pó de madeira) e lixação (pó de madeira).

Quanto às formas de acondicionamento destes resíduos, identificadas nas 11 fábricas, predominam:

a) caçambas e tambores (aparas e cavacos de madeira);

b) silos (serragem e pó de madeira).

O acondicionamento nos silos se faz por meio de sistemas de exaustão, onde tubulações flexíveis são conectadas diretamente às máquinas, realizando o transporte destes resíduos até o silo, normalmente localizadas externamente ao galpão de produção. Desta forma, diferentemente dos resíduos de aparas, os resíduos de madeira na forma de serragem e pó não são segregados, imprimindo a estes características físicas e químicas compostas pelas diversas matérias-primas de origem (madeira maciça e painéis crus ou revestidos).

De acordo com o resultado apresentado na Tabela 2, é gerada mensalmente, nas 11 fábricas de móveis inventariadas, uma média de 1.699 toneladas de resíduos de madeira. Isso corresponde a uma geração média diária de 77 toneladas, considerando 22 dias/mês de produção.

As características físicas (umidade e densidade aparente), teor de cinzas e poder calorifico dos resíduos são apresentados na Tabela 3. As densidades aparentes reforçam a vantagem competitiva do reaproveitamento dos resíduos na forma de briquetes em comparação às formas de aparas, serragem e pó, uma vez que a densidade dos briquetes de madeira e seus derivados chegam a $1.200 \mathrm{~kg} / \mathrm{m}^{3}$, aproximadamente 5 a 6 vezes a densidade dos resíduos amostrados. Esse ganho se reflete na redução dos custos de transporte e armazenagem dos resíduos, concentrando muito mais energia por volume de material.

TABELA 2: Geração mensal de resíduos de madeira nas 11 fábricas inventariadas.

TABLE 2: Monthly production of wood wastes in 11 mills.

\begin{tabular}{|c|c|c|c|c|c|c|c|c|c|c|c|c|}
\hline \multirow{3}{*}{ Resíduo } & \multicolumn{11}{|c|}{ Empresas amostradas / Quantidade de resíduos gerados ( $\mathrm{t} / \mathrm{mês}$ ) } & \multirow{3}{*}{ Total } \\
\hline & \multirow{2}{*}{$\begin{array}{c}\text { Micro } \\
1\end{array}$} & \multicolumn{4}{|c|}{ Pequena } & \multicolumn{5}{|c|}{ Média } & \multirow{2}{*}{$\begin{array}{c}\text { Grande } \\
11\end{array}$} & \\
\hline & & 2 & 3 & 4 & 5 & 6 & 7 & 8 & 9 & 10 & & \\
\hline & 0,16 & 13,5 & 17,55 & 9,23 & 32,4 & 13,5 & 5,6 & 20,3 & 20,3 & 81 & 112,5 & 326 \\
\hline 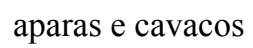 & 0,75 & 45 & 54 & 17,25 & 111 & 12 & 97,5 & 330 & 120 & 412,5 & 172,5 & 1.373 \\
\hline Total & 0,91 & 58,5 & 71,55 & 26,48 & 143,4 & 25,5 & 103,1 & 350,3 & 140,3 & 493,5 & 285 & 1.699 \\
\hline
\end{tabular}

TABELA 3: Valores médios de densidade aparente, umidade, poder calorífico e teor de cinzas dos resíduos. TABLE 3: Average values of apparent density, moisture, caloric power and ash content of residues.

\begin{tabular}{lcccc}
\hline \multicolumn{1}{c}{ Resíduos } & $\begin{array}{c}\text { Densidade } \\
\mathrm{aparente}^{1}(\mathrm{~kg} / \\
\left.\mathrm{m}^{3}\right)\end{array}$ & $\begin{array}{c}\text { Umidade }{ }^{2} \\
(\%)\end{array}$ & $\begin{array}{c}\text { Poder }^{2} \\
\text { Calorífico }^{2} \\
(\mathrm{kcal} / \mathrm{kg})\end{array}$ & $\begin{array}{c}\text { Teor de } \\
\text { Cinzas }^{2} \\
(\%)\end{array}$ \\
\hline $\begin{array}{l}\text { A1-mistura de resíduos de painéis de MDF, aglomerado, } \\
\text { compensado s/ revestimento e madeira }\end{array}$ & 209 & 7,5 & 4632 & 0,97 \\
A2-resíduo de madeira maciça & 200 & 16,5 & 4730 & 0,48 \\
A3-resíduo de MDF (medium density fiberboard) & 303 & 10,3 & 4732 & 0,73 \\
A4-mistura de resíduos de painéis de MDF, aglomerado e & 216 & 10 & 4633 & 2,58 \\
compensado com e s/ revestimento & & & & \\
A5-mistura de resíduos de painéis de MDF, aglomerado e & 289 & 9,2 & 4095 & 5,02 \\
compensado s/ revestimento & 279 & 3,4 & 4316 & 30,3 \\
A6-pó fino (lixação de painéis) & - & 17,8 & 4259 & 0,14 \\
\hline B1-madeira maciça & - & 22,7 & 4419 & 0,79 \\
B2-compensado & - & 9,8 & 4523 & 0,27 \\
B3-MDF & - & 10,3 & 4527 & 0,33 \\
B4-aglomerado cru & - & 9,8 & 4411 & 0,95 \\
B5-aglomerado BP & - & 10,1 & 4301 & 0,40 \\
B6-aglomerado FF & & & & \\
\hline
\end{tabular}

Em que: ${ }^{1}=$ valores médios de 5 repetições; ${ }^{2}=$ valores médios de 3 repetições. 
A viabilidade técnica e econômica para o reaproveitamento energético dos resíduos de madeira e derivados, especialmente na forma de briquetes, tem relação com as características de umidade e poder calorífico dos resíduos. Cabe destacar ainda a importância do teor de cinzas e densidade aparente, para os estudos de disposição final dos rejeitos da queima dos briquetes e logística de transporte.

De acordo com Quirino (1991), a umidade ideal do resíduo lignocelulósico para a produção de briquetes gira em torno de 8 a $12 \%$. Deste modo, verifica-se, na Tabela 3 , a predominância desta faixa para os resíduos dos painéis reconstituídos, sugerindo maior competitividade econômica aos briquetes, visto não haver a necessidade de secagem dos resíduos antes do seu processamento. Essa característica vem a contribuir com a redução dos custos de investimento e operação, incrementando o lucro do empreendimento. Estes dados se tornam ainda mais expressivos quando se verifica a tendência de substituição da madeira maciça pelos painéis na fabricação de móveis. Identificou-se, no estudo, que o consumo de aglomerado e MDF nas 11 fábricas amostradas correspondem a $46 \%$ e $24 \%$, respectivamente, em relação ao total de matériaprima à base de madeira utilizada, sugerindo uma maior geração destes resíduos.

O elevado poder calorífico dos resíduos, conforme verificado na Tabela 3, contribui para a viabilidade econômica da proposta do presente estudo. Os briquetes apresentam uma densidade aparente aproximadamente 5 vezes superior à dos resíduos in natura, concentrando muito mais calor por volume, além de reduzir os custos com transporte.

Quanto ao teor de cinzas, de acordo com a Tabela 3, verificou-se que os resíduos que apresentaram as maiores concentrações pertencem ao Grupo A, com destaque para a amostra A6, composta por resíduos gerados a partir da lixação dos painéis sem revestimento (cru). Estes painéis recebem uma aplicação de massa seladora no preparo das superfícies e laterais para a etapa de pintura. Posteriormente, os painéis são lixados, para a retirada do excesso da massa, gerando um rejeito composto por resíduos de madeira, massa seladora e abrasivos da lixa.

A preocupação com o teor de cinzas relaciona-se com a disposição final dos rejeitos da queima de biomassa (briquetes). Assim, quanto menor a produção de cinzas, menores os custos com o seu tratamento e/ou disposição final. A fim de se avaliar a possibilidade de disposição das cinzas no solo, sem comprometer a qualidade e características naturais do meio, procedeu-se à sua classificação nas amostras de resíduos dos grupos A e B.

A Tabela 4 apresenta os resultados das análises dos parâmetros inorgânicos no lixiviado das cinzas geradas a partir da queima dos resíduos amostrados. Observou-se que as concentrações encontradas são inferiores aos limites padrões preconizados pela NBR 10004 (ABNT, 2004), sendo, portanto, classificados como não perigosos (Classe II), indicando que podem ser dispostas no solo, com exceção das amostras de cinza dos resíduos A4 e B6, que apresentaram concentrações de cromo acima do limite preconizado, ou seja, $5 \mathrm{mg} . \mathrm{l}^{-1}$. Assim, as cinzas dos resíduos A4 e B6 foram classificadas como resíduo perigoso (Classe I). A presença do cromo nestas cinzas pode estar associada aos resíduos de revestimento dos painéis $\mathrm{BP}$ e FF.

Quanto à concentração dos compostos orgânicos avaliados (VOC, SVOC e Formaldeído) nos lixiviados dos resíduos, somente o clorofórmio e o formaldeído foram detectados (Tabela 5).

$\mathrm{O}$ clorofórmio apresentou valores muito inferiores ao limite preconizado pela NBR 10004 para resíduos Classe 1 (ABNT, 2004), ou seja, $6 \mathrm{mg} \mathrm{l}^{-1}$. Quanto ao formaldeído, apesar da Norma não especificar nenhum valor como limite padrão, este é classificado como potencialmente cancerígeno pela Agência Internacional de Pesquisa do Câncer (IARC, 2006). Conforme esperado, a amostra B1, composta por madeira maciça, não apresentou níveis significativos de formaldeído. As amostras A6, pó de lixação de painéis, também não apresentaram teores significativos de formaldeído, possivelmente devido ao fato de se tratar de resíduos superficiais dos painéis, onde não se encontram resquícios de cola.

Desta forma, as concentrações encontradas nos lixiviados dos resíduos sugerem a necessidade de uma investigação das concentrações de formaldeído nos gases resultantes da queima destes resíduos, estado físico em que este composto apresenta maiores riscos à saúde humana.

Considerando os parâmetros orgânicos e inorgânicos preconizados pela NBR 10.004, os resíduos analisados, com exceção das cinzas geradas na combustão dos resíduos A4 e B6, foram classificados como Classe II (não perigosos). Este resultado comprova, de forma mais delineada, os resultados encontrados por Rezende et al. (2006), 
TABELA 4: Análise dos parâmetros inorgânicos nos lixiviados dos resíduos e de suas respectivas cinzas.

TABLE 4: Wood residues inorganic parameters and their respective ash analyses.

\begin{tabular}{|c|c|c|c|c|c|c|c|}
\hline \multicolumn{2}{|c|}{ Parâmetros } & Bário & Arsênio & Chumbo & Selênio & Cádmio & Cromo \\
\hline \multicolumn{2}{|c|}{ Padrão } & 70 & 1 & 1 & 1 & 0,5 & 5 \\
\hline \multirow{12}{*}{ 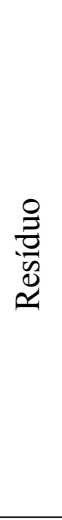 } & A1 & 0,877 & nd & 0,018 & 0,006 & 0,020 & 0,025 \\
\hline & A2 & 0,115 & nd & 0,001 & 0,015 & 0,014 & 0,002 \\
\hline & A3 & 0,093 & nd & 0,008 & 0,003 & 0,017 & 0,001 \\
\hline & A4 & 0,319 & nd & 0,001 & 0,008 & 0,006 & 0,002 \\
\hline & A5 & 0,148 & nd & 0,007 & 0,004 & 0,007 & 0,011 \\
\hline & A6 & 2,581 & nd & nd & 0,114 & nd & 0,012 \\
\hline & B1 & 0,127 & nd & 0,007 & 0,022 & 0,020 & nd \\
\hline & B2 & 0,035 & nd & 0,002 & 0,005 & 0,005 & nd \\
\hline & B3 & 0,155 & nd & 0,008 & 0,006 & 0,007 & nd \\
\hline & B4 & 0,023 & nd & 0,007 & nd & 0,005 & nd \\
\hline & B5 & 0,093 & nd & 0,008 & 0,005 & 0,001 & nd \\
\hline & B6 & 0,004 & nd & 0,030 & nd & 0,004 & 0,028 \\
\hline \multirow{12}{*}{$\stackrel{\mathscr{\Xi}}{\overparen{U}}$} & A1 & 0,111 & nd & nd & 0,021 & 0,002 & 3,385 \\
\hline & A2 & 0,440 & nd & 0,008 & 0,002 & 0,005 & 0,009 \\
\hline & A3 & 0,017 & nd & nd & 0,066 & nd & 0,991 \\
\hline & A4 & 0,105 & nd & nd & 0,109 & 0,001 & 16,466 \\
\hline & A5 & 0,303 & nd & 0,008 & nd & 0,008 & 0,009 \\
\hline & A6 & 0,164 & nd & 0,006 & nd & 0,009 & 0,002 \\
\hline & B1 & 0,353 & nd & 0,016 & nd & 0,010 & 0,017 \\
\hline & B2 & A & A & A & A & A & A \\
\hline & B3 & 0,426 & nd & nd & 0,539 & 0,026 & 2,164 \\
\hline & B4 & 1,093 & nd & nd & 0,054 & 0,001 & 3,890 \\
\hline & B5 & 0,99 & nd & 0,002 & nd & 0,005 & 4,858 \\
\hline & B6 & 0,574 & 0,008 & nd & 0,077 & nd & 83,476 \\
\hline
\end{tabular}

Em que: ${ }^{\mathrm{A}}=$ Análise não realizada; nd = não detectado.

TABELA 5: Concentrações de formaldeído e clorofórmio nos resíduos.

TABLE 5: Formaldehyde and chloroform concentration at wood residues.

\begin{tabular}{cccccccc}
\hline \multicolumn{7}{c}{ Concentrações (mg..$^{-1}$ ) } \\
\hline Parâmetro & B1 & B2 & B3 & B4 & B5 & B6 & A6 \\
\hline Formaldeído & 0,19 & 12,65 & 12,92 & 12,93 & 12,73 & 8,96 & 0,15 \\
Clorofórmio & nd & 0,014 & 0,017 & 0,019 & 0,018 & 0,016 & 0,011 \\
\hline
\end{tabular}

Em que: nd = não detectado.

em estudo sobre os resíduos de madeira e derivados gerados nas fábricas de móveis do mesmo Polo Moveleiro (Ubá - MG), e por Kozak et al. (2008) e Lima (2005), ao realizarem um diagnóstico em empresas de móveis de madeira situadas no Polo Moveleiro de Arapongas, Paraná.

A Tabela 6 apresenta os resultados das análises dos gases gerados no processo de queima dos resíduos de madeira. B1 (madeira maciça), B3 (MDF) e B5 (Aglomerado BP).

As análises dos gases apresentaram concentrações de $\mathrm{NO}_{x}$ e $\mathrm{CO}$ dentro dos limites estabelecidos pela Resolução CONAMA 382/2006 (CONAMA, 2006). Quanto ao clorofórmio e formaldeído, devido à ausência de padrões para a referida Resolução, adotou-se como referência a instrução técnica alemã TA LUFT de $27 / 02 / 1986$. Esta norma estabelece como limite máximo a concentração de $100 \mathrm{mg} / \mathrm{Nm}^{3}$, tanto para o clorofórmio quanto formaldeído. $\mathrm{O}$ maior valor de concentração encontrado de formaldeído no resíduo de $\mathrm{MDF}$ foi de $8,22 \mathrm{mg} / \mathrm{Nm}^{3}$. Assim, de acordo com os parâmetros avaliados e nas condições desenvolvidas neste trabalho, não há impedimento ao reaproveitamento energético dos resíduos de madeira e derivados das 11 fábricas de móveis amostradas no que tange à emissão de gases. 
TABELA 6: Análise dos gases da queima dos resíduos.

TABLE 6: Gas analysis after wood residue incineration.

\begin{tabular}{lccccc}
\hline Parâmetros & \multicolumn{5}{c}{ Concentrações médias (mg/Nm $\left.{ }^{3}\right)$} \\
& madeira maciça (B1) & aglomerado BP (B5) & $\begin{array}{c}\text { aglomerado } \\
\text { MDF (B3) }\end{array}$ & $\begin{array}{c}\text { VMP } \\
\text { CONAMA382/06 }\end{array}$ & $\begin{array}{c}\text { VMP }^{\mathrm{A}} \\
\text { TA LUFT }\end{array}$ \\
\hline Formaldeído & 4,85 & 1,96 & 8,22 & - & 100 \\
Clorofórmio & nd & $<0,0001$ & nd & - & 100 \\
$\mathrm{NO}_{\mathrm{x}}$ & 55,73 & 237 & 250 & 650 & - \\
$\mathrm{CO}_{2}$ & 655 & 652 & 747 & - & - \\
$\mathrm{CO}$ & 17,29 & 17,29 & 17,29 & $1.300^{\mathrm{B}}$ & - \\
\hline
\end{tabular}

Em que: ${ }^{\mathrm{A}}=$ Valor máximo permitido; ${ }^{\mathrm{B}}=$ Valor mais restritivo; $\mathrm{nd}=$ não detectado.

\section{CONCLUSÕES E RECOMENDAÇÕES}

Os resíduos de madeira da indústria moveleira do Polo de Ubá apresentaram um potencial para o reaproveitamento energético relacionado aos seus altos valores de poder calorifico.

Os resíduos e as respectivas cinzas geradas após queima foram classificados como resíduos Classe 2, não perigosos. No entanto, cuidados devem ser tomados para a disposição final das cinzas dos resíduos constituídos por painéis de MDF, aglomerado e compensado com e sem revestimento (resíduo A4) e aglomerado FF (resíduo B6), que deverão ser encaminhados a aterros industriais Classe I, devido à presença de cromo acima dos limites preconizados pela norma brasileira.

Os gases gerados a partir da queima dos resíduos na forma como se encontram nas fábricas não apresentaram concentrações de poluentes acima dos padrões preconizados pelo CONAMA. Salienta-se que os valores das concentrações de formaldeído nos gases, embora não determinados pelo CONAMA, apresentaram valores inferiores aos valores máximos permitidos por norma alemã para controle da poluição do ar, usada como referência neste estudo.

\section{AGRADECIMENTOS}

Os autores agradecem ao Serviço Brasileiro de Apoio às Micro e Pequenas Empresas (SEBRAE) e à Financiadora de Estudos e Projetos (FINEP), pelo apoio financeiro; ao Sindicato Intermunicipal das Indústrias de Marcenaria de Ubá (INTERSIND) e às fábricas de móveis: Sier Móveis, Cedrus Movelaria Ltda., Móveis Tradição Ltda., Mavaular Móveis Ltda., Paropas, Móveis Bettio Ltda., Indústria de Móveis Paschoalino Ltda., Mademóveis Indústria e Comercio Ltda., Cel Móveis Ltda., Móveis Novo
Horizonte Ltda. e Móveis Perini, pela parceria no desenvolvimento deste estudo.

\section{REFERÊNCIAS BIBLIOGRÁFICAS}

ABIMOVEL. Associação Brasileira das Indústrias do Mobiliário. Panorama do Setor Moveleiro no Brasil. São Paulo, 2005. Disponível em: $<\mathrm{http} / / /$ www.abimovel.com.br>.Acesso em junho de 2008. ABNT. Associação Brasileira de Normas Técnicas. Conjunto de Normas sobre resíduos. NBR 10004, NBR 10005, NBR 10006, NBR 10007. Resíduos Sólidos. Classificação. Procedimentos. Amostragem. Rio de Janeiro: ABNT, 2004.

NBR 10700: Planejamento de amostragem em dutos e chaminés de fontes estacionárias. Rio de Janeiro: ABNT, 1989.

NBR 10701: Determinação de pontos de amostragem em dutos e chaminés de fontes estacionárias. Rio de Janeiro: ABNT, 1989.

NBR 10702: Efluentes gasosos em dutos e chaminés de fontes estacionárias - Determinação da massa molecular. Rio de Janeiro: ABNT, 1989.

NBR 11966: Efluentes gasosos em dutos e chaminés de fontes estacionárias - Determinação da velocidade e vazão. Rio de Janeiro: ANBT, 1989.

NBR 11967: Efluentes gasosos em dutos e chaminés de fontes estacionárias - Determinação da umidade. Rio de Janeiro: ABNT, 1989.

NBR 12019: Efluentes gasosos em dutos e chaminés de fontes estacionárias - Determinação de material particulado. Rio de Janeiro: ABNT, 1990.

NBR 12020: Efluentes gasosos em dutos e chaminés de fontes estacionárias - Calibração dos equipamentos utilizados em amostragem Método de Ensaio. Rio de Janeiro: ABNT, 1992. NBR 8289: Determinação do teor de 
cinza. Rio de Janeiro: ABNT, 1986.

. NBR 8293: Determinação de umidade.

Rio de Janeiro: ABNT, 1986.

NBR 8969: Definição dos termos empregados no estudo de poluição do ar. Rio de Janeiro: ABNT, 1985.

Resolução 382/2006. Estabelece limites máximos de emissão de poluentes atmosféricos para fontes fixas. Brasília, 2006.

ABRELPE. Associação Brasileira de Empresas de Limpeza Pública e Resíduos Especiais. Panorama dos Resíduos Sólidos no Brasil. São Paulo, 2007. Disponível em www.abrelpe.org.br. Acesso em janeiro de 2009.

ABREU, L. B.; MENDES, L. M.; SILVA, J. R. M. Aproveitamento de resíduos de painéis de madeira gerados pela indústria moveleira na produção de pequenos objetos. Revista Árvore, Viçosa, v. 33, n. 1, p. 171-177, 2009.

American Society for Testing Materials - ASTM D-2015-66. Gross Calorific Value of Solid Fuel by the Adiabatic Bomb Calorimeter.1972.

Analytical Solutions S.A. Norma interna: PE 49-127 Rev.6 Cromatografia Gasosa acoplada a espectrômetro de massa. Baseada na Environmental Protection Agency - EPA, Method 030.

CETESB. Companhia de Tecnologia de Saneamento Ambiental. Efluentes gasosos em dutos e chaminés de fontes estacionárias - Amostragem e determinação de Óxidos de Nitrogênio. São Paulo, 1992.

CONAMA. Conselho Nacional do Meio Ambiente.

Resolução 313/2002. Dispõe sobre o Inventário Nacional de Resíduos Sólidos Industriais. Brasília, 2002.

Environmental Protection Agency - EPA. EPA Method 030. Volatile Organic Sampling Train for Volatiles. 1986.

Environmental Protection Agency - EPA. EPA Method 8315A. Determination of carbonyl compounds by high performance liquid chromatography. 1996.

FIEMG. Federação das Indústrias de Minas Gerais. Ubá Móveis de Minas - Arranjo Produtivo Local de Ubá. Ubá - MG, 2008. Disponível em: http://www. fiemg.org.br/apluba. Acesso em junho de 2008. German Environment Ministry. TA Luft - Technical
Instructions on Air Quality Control. 2002.

IARC. International Agency for Research on Cancer. IARC Monographs on the evaluation of carcinogenic risks to humans. Formaldehyde, 2-Butoxyethanol and 1-tert-Butoxy- 2-propanol. v. 88. Lyon, 2006. Disponívelem: http://monographs. iarc.fr/ENG/Monographs/vol88/volume88.pdf. Acesso em março de 2009.

KOZAK, P. A. et al. Identificação, Quantificação e Classificação dos Resíduos Sólidos de uma Fábrica de Móveis. Revista Acadêmica, Ciência Agrária e Ambiental, Curitiba, v. 6, n. 2, p. 203-212, 2008. LIMA, E. G. Diagnóstico Ambiental de Empresas de Móveis em Madeira Situadas no Pólo Moveleiro de Arapongas - PR. Curitiba: UFP, 2005. Dissertação (Mestrado em Engenharia Florestal)-Universidade Federal do Paraná, 2005.

PIRES, V. A. V. et al. Viabilidade Econômica de Implantação da Central de Gerenciamento de Resíduos no Pólo Moveleiro de Ubá - MG. Cerne, Lavras, v.14, n. 4, p. 295-303, 2008.

QUIRINO, W. F. Biomassa ganha espaço e já é terceira principal fonte. BBC Brasil, Tecnologia e Saúde, 2002. Disponível em: http://www.bbc. co.uk/portuguese/ciencia/020814_energia5ae. shtml. Acesso em:junho de 2008.

QUIRINO, W. F. Briquetagem de Resíduos Ligno-Celulósicos. Circular Técnica do LPFLaboratório de Produtos Florestais do IBAMA, Brasília, v. 1, n. 2, p. 69-80, 1991.

REZENDE, A.A. P.et al. Proposta de Gerenciamento Integrado dos Resíduos Sólidos em Pólo Moveleiro .In: SIMPÓSIO ÍTALO BRASILEIRO DE ENGENHARIA SANITÁRIA E AMBIENTAL, 8., 2006, Fortaleza. Anais... Rio de Janeiro: ABES, 2006.

Rio de Janeiro, 2006.

SCHNEIDER, V. E. et al. Gerenciamento Ambiental na Indústria Moveleira - estudo de caso no município de Bento Gonçalves. In: ENCONTRO DE ENGENHARIA DE PRODUÇÃO, 23., 2003, Ouro Preto. Anais... Encontro Nacional de Engenharia de Produção - ENEPRO, 2003.

SILVA, C. M. et al. Relatório Final: Proposta de Gerenciamento Integrado dos Resíduos Sólidos do Pólo Moveleiro de Ubá - MG. Viçosa: UFV, 2005. Não Publicado. 